\title{
Die Bewegungen des Auges, veranschaulicht durch das Phaenophthalmotrop.
}

\author{
Von \\ F. C. Donders.
}

Hierzu Tafel II.

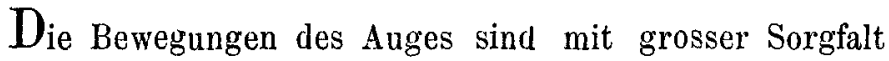
untersucht und ihr ziemlich verwickelter Mechanismus dadurch im Allgemeinen aufgeklärt worden. Wir kennen die Gesetze, nach welchen die Bewegungen Statt haben und wissen ebenfalls unter welchen Umständen gewisse, wenn auch geringe und für verschiedene Augen nicht ganz übereinstimmende $A b$ weichungen dieser Gesetze volkommen. Allein trotz dieser verhältnissmässigen Vollkommenheit unserer Kenntniss ist die Lehre von den Augenbewegungen für viele Ophthalmologen ein Stein des Anstosses geblieben. Die Literatur ist, besonders was die sogenannte Raddrehung betrifft, nicht von Verwirrung frei zu sprechen und man sieht bei den Vorlesungen, wie die angestrengtesten Versuche seinen $\mathrm{Zu}-$ hörern einen deutlichen Einblick in den genannten Mechanismus beizubringen, bei gar Vielen Schiffbruch leiden.

Man hat nun versucht, dem Vorstellungsvermögen durch gewisse Instrumente zu Hülfe zu kommen, die man Ophthalmotropen genannt hat. Dieselben haben im Allgemeinen den Zweck, die Wirkung der Augenmuskeln anschaulich zu machen. Aber es kommt zuerst darauf an, dass man sich von den Bewegungen selbst eine rich- 
tige Vorstellung bilde. Hierin scheint für die Meisten die Hauptschwierigkeit zu liegen. Stehen ihnen aber die Bewegungen klarer vor Augen, dann begreifen sie sofort, welche Muskeln dabei activ verkürzt, welche passiv verlängert werden. Es erschien deshalb wünschenswerth, ein Instrument zu construiren, welches vorzugsweise die Bewegungen veranschaulicht. Von den schon existirenden Ophthalmotropen möge es durch die Bezeichnung ,Phaen-

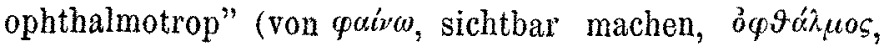

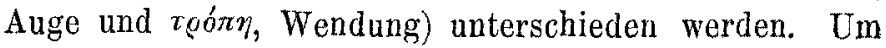
seine Anwendung klar zu inachen, werden wir, zugleich mit der Beschreibung des Instruments, die Augenbewegungen näher verfolgen.

Es gab eine Zeit, wo man von den Augenmuskeln ausging und aus ihnen Schlüsse zog bezüglich der Bewegungen des Bulbus. Man hielt die vier geraden Augenmuskeln für zureichend, um der Fixationslinie, die durch den Drehpunkt und den im Raum fixirten Punkt geht, jede gewünschte Richtung $2 u$ geben und man meinte, dass hiermit allen Anforderungen genügt sei. Für die schrägen Augenmuskeln musste mithin eine andere Wirkung aufgesucht werdeu. Man fand zwei für eine. Die Ursache des Accommodationsvermögens war nicht bekannt: sollten nun die schrägen Augenmuskeln nicht im Stande sein, durch Druck auf den Bulbus die Gesichtsaxe zu verlängern und solcherweise für die Accommodation zu sorgen? Ohne jeglichen Beweis gab man sich mit dieser gegenseitigen Auflösung zweier Unbekannten zufrieden. Die Muskeln wirkten hierbei dann gemeinschaftlich. Aber auch der besonderen Wirkung wusste man ihre Aufgabe anzwweisen. Aus der Richtung der schrägen Muskeln folgerte man, dass sie im Stande sein müssten, das Auge um die Sehaxe zu drehen, und eine derartige Drehung meinte Hueck bei Seitwärtsneigung des Kopfes nun wirklich demonstriren zu können: bis zu 
einer Ausdehnung von $25^{\circ}$ bis $28^{\circ}$ werde die Neigung des Kopfes jederseits durch eine Drehung um die Gesichtsaxe compensirt, so dass die verticalen Meridiane nicht aufhörten, vertical zu bleiben. Die erste, den schrägen Augenmuskeln zuerkannte Rolle verfiel von selbst. bei der Entdeckung. der Ursache der Accommodation, und die Drehung um die Gesichtsaxe war nicht haltbar bei der leicht zu constatirenden Thatsache, dass, bei Neigung des Kopfes nach einer Seite, die Nachbilder auf der Netzhaut in gleichem Sinne und gewiss ungefähr in gleichem Maasse, ihre Richtung veränderten. Den glücklichen Gedanken. zur Beurtheilung des Augenstandes die Nachbilder zu consultiren, verdanken wir Ruete, der nun auch bald den schrägen Muskeln ihre wahre Bedeutung zuzuweisen wusste. Es ergab sich nämlich, dass der verticale Meridian seine verticade Stellung behält, wenn das Auge entweder um die Queraxe gerade nach oben und nach unten, oder um die verticale Axe in einer horizontalen Fläche nach rechts und links bewegt wird, dass dagegen, wenn das Auge seitwärts nach oben gerichtet, der verticale Meridian nach derselben Seite, bei der Richtung seitwärts nach unten, nach der entgegengesetzten Seite hinüberneigt. Nun war es klar, dass, sobald beim Sehen gerade nach oben und nach unten die $\mathrm{mm}$. recti superior. und inferior., deren Richtung einen Winkel von ungefähr $20^{\circ}$ mit der Gesichtsaxe bildet, allein thätig waren, der verticale Meridian dabei eine schräge Stellung annehmen musste, die allein compensirt werden kann durch Zusammenwirken des m. obliquus inferior mit dem rectus superior und von dem $m$. obliquus superior mit dem rectus inferior. In beiden Fällen unterstützen zwei zusammenwirkende Muskeln einander auf der transversalen Axe, um auf der Sehaxe sich gegenseitig aufzuheben und so allein kann der verticale Meridian bei der Richtung der Fixations- 
linien gerade nach oben und gerade nach unten seinen verticalen Stand beibehalten.

Es wird, wie man sieht, bei dieser Methode zuvörderst die Bewegung des Auges festgestellt, um daraus auf die Muskelwirkung zu schliessen. Dies ist, wie ich besonders hervorhob, der einzige Weg, um den Mechanismus einer Bewegung aufzuklären. An die Beschreibung eines Muskels mag der Anatom die Frage knüpfen, welche Bewegung aus seiner Contraction resultiren würde, wenn sie wirklich isolirt vorkäme, - die Aufgabe des Physiologen aber ist es, die Bewegungen selbst zu untersuchen, um darnach zu bestimmen, welche Muskeln bei dem neuen Gleichgewichtszustande verkürzt oder verlängert sind und welcher Spannung sie dabei unterworfen sind. Hueck glaubte sich von dem Bestehen der Raddrehung beim Seitwärtsneigen des Kopfes überzeugt zu haben aus der veränderten Richtung der sichtbaren Bindehaut. gefässe. Ich wandte dagegen ein, dass, bei den Hueckschen Versuchen, die Fixationslinien, um einen und denselben nahegelegenen Punkt fortgesetzt zu fixiren, ihre relative Richtung zum Kopf veränderten und dass die hierbei entstehende Neigung der verticalen Meridiane eine Raddrehung simuliren könne. Ganz entscheidend war der Versuch, bei welchem das Auge sich in einem zwischen den Zähnen gehaltenen kleinen Spiegel selbst beschaute und nun bei jeder Kopfbewegung die Bindehautgefässe, und auf der Iris sichtbare Punkte unverändert dieselbe relative Lage zu den Augenlidern und -Winkeln, und zu Strichen im Gesicht, behalten suh. Ich überzeugte mich ferner, indem ich von den Nachbildern eines verticalen Bandes Gebrauch machte, dass, für jede bestimmte Richtung der Fixationslinie relativ zur verticalen Kopfstellung und wie auch immer diese Richtung zu Stande gekommen sein mochte, - die Stellung 
des verticalen Meridians und damit die des ganzen Auges stets unveränderlich dieselbe war.

Das hiermit gefundene Gesetz formulirt Helmholtz, der es das Donders'sche Gesetz nennt, folgendermaassen: „Der Raddrehungswinkel jedes Auges ist bei parallelen Blicklinien eine Function nur ron dem Erhebungswinkel und dem Seitenwendungswinkel."

Man sieht, dass Helmholtz zur Bestimmung der Stellung des Auges einen Raddrebungswinkel einführt. Später wird uns klar werden, was Helmholtz hierunter versteht. Ich selbst glaubte den Ausdruck vermeiden zu müssen, da ich die Raddrehung nicht für bewiesen hielt, - wie denn auch, nach dem Listing'schen Gesetz, beim Uebergang aus der primären in die secundäre Stellung, gleichgültig welche, von einer wahren Raddrehung des Auges nicht die Rede sein kann. Es schien mir, dass mit der Neigung des ursprünglich verticalen Meridians, bei aufrechter Kopfhaltung, die stellung des Auges ebenso gut und in Uebereinstimmung mit der Richtung der Nachbilder, bestimmt würde. Ich kam in meiner Arbeit zu dem Resultat, dass sich die verticalen Meridiane um so stärker neigen, je mehr, bei gleicher Hebung oder Senkung, der Blick seitwärts gerichtet und je mehr, bei gleicher seitlicher Abweichung, der Blick aufwärts oder abwärts bewegt wird. Ich machte später, nach der in der Arbeit genau beschriebenen Methode*) eine Anzahl Bestimmungen der, jeder Richtung der Fixationshinie entsprechenden Abweichung der verticalen Stellung; allein, da es mir nicht gelang, diese Abweichungen auf ein bestimmtes Gesetz zurückzuführen, so blieb der zweite Theil meiner Arbeit unvollendet. Andere waren in ihren Bemühungen nicht glücklicher als ich.-

*) Holländische Beiträge zu den anatomischen und physiologischen Wissenschaften, 1846 . B. 1. S. 135. 
Aber siehe da! ein Gesetz wurde a priori ausgesprochen und nun war es nicht so schwer, dasselbe an den Beobachtungen zu prifen: es wurde probehaltig erfunden.

Das Princip, durch Listing aufgestellt*), lautet so: „Aus der normalen (primären) Stellung wird das Auge in irgend eine andere, secundäre, in der Weise versetzt dass man sich diese Versetzung als das Resultat einer Drehung um eine bestimmte Drehungsaxe vorstellen kann, welche jederzeit, durch das Augencentrum gehend, auf ìer primären und der secundären Richtung der optischen Axe zugleich senkrecht steht, sodass also jede secundäre Stellung des Auges zur primären in der Relation steht, vermöge welcher die auf die optische Axe projicirte Drehung = 0 wird."

Meissner fand die Resultate seiner Untersuchungen mit diesem Gesetz in Uebereinstimmung; jedoch haben wir wieder Helmholtz den einfachen Versuch zu danken, wodurch sich Jeder von dessen Gültigkeit überzeugen kann. Dieser Versuch beruht auf dem Gebrauch der Nachbilder. Wir haben oben gesehen, dass das Nachbild eines verticalen Bandes vertical bleibt, sobald wir bei aufrechter Kopf haltung unseren Blick in einer verticalen Ebene, also in der Richtung des Bandes, aufund nieder bewegen. Dies nun gilt nicht allein für ein verticales Band, sondern auch für ein Band in jeder Richtung, einerlei welcher: man hat nur das Auge so zu bewegen, dass die Fixationslinie und das ausgespante Band in derselben Ebene liegen. Macht man Strahlen auf einer Wand, die von einem Mittelpunkt ausgehen, um welchen ausserdem ein hellfarbiges Band drehbar ist, so kann man das letztere der Reihe nach mit jedem der Strahlen zusammenfallen lassen und jedesmal wird man,

*) Zuerst mitgetheilt durch Ruete. Lehrbuch der Ophthalmologie. 2. Auft. Braunschweig, 1853. Bd. 1. S. 36 . 
nach Fixation des Bandes bei aufrechter Kopfhaltung, das Nachbild dem Strahle folgen sehen, sobald sich der Blick in dessen Richtung fortbewegt, - bei jeder Bewegung über einen der anderen Strahlen aber hiervon abweichen sehen. Darin liegt nun der Beweis für die Richtigkeit des Listing'schen Gesetzes klar vor Augen. Der Versuch lehrt, dass der Meridian, in welchem das Band liegt, seine Richtung behält, wenn die Fixationslinie in der Ebene dieses Meridians fortschreitet. Der Meridian dreht sich also in diesem Falle um eine Axe, die ihn lothrecht schneidet und zwar im Drehpunkt. Mit anderen Worten, - das Auge, indem es aus der primären in die secundäre Stellung übergeht, dreht sich um eine Axe, welche senkrecht steht auf der Ebene, in welcher die primäre und secundäre Fixationslinie liegt: das ist das Listing'sche Gesetz.

Dieses Gesetz muss nun zuvörderst durch das Phaenophthalmotrop anschaulich gemacht werden.

An diesem Instrument (in Fig. 1 perspectivisch von der Seite gesehen) dreht sich der Augapfel 00 mit dem Ringe $R R$ (worüber später) in dem Ringe $R^{\prime}$ und $z$ war in der Stellung der Figur um eine horizontale Axe, deren eines Ende bei a' sichtbar ist. So bewegt sich also die Fixationslinie in einer senkrechten Ebene nach oben und nach unten. Mit der Axe $a^{\prime}$ ist ein rundes Plättchen verbunden, welches mit einem Gradbogen $g^{d}$ versehen ist; der Index $i^{\prime}$, welcher in der Figur auf $0^{0}$ steht, ist in dem Ringe $R^{\prime}$ mit zwei Schrauben $s^{\prime}$ befestigt. Dreht sich nun der Bulbus um die Axe $a^{\prime}$, so liest man auf $g^{\prime}$ das Maass dieser Drehung in Graden ab.

In dem vor dem Bulbus befindlichen Ring $\mathbf{r}$ können zwei dünne Stäbchen $\mathrm{kk}$ eingeschraubt werden; sie sind mit dem Ring $r$ um die Fixationslinie drehbar und der Index $i^{0}$ zeigt auf dem Gradbogen $g^{0}$ an, wie weit sie gedreht sind. In der Stellung des Instruments entspricht 
die verticale Richtung der Stäbchen dem Punkte $0^{\circ}$. Diese Stäbchen vergegenwärtigen den verticalen Meridian. Dreht sich das Auge um die Queraxe a', so behält der Meridian seine verticale Stellung. So bewegt sich die Fixationslinie des Auges in einer verticalen Ebene bei der Bewegung gerade nach oben und gerade nach unten. Dies bedarf keiner näheren Erläuterung.

Man kann nun dem Instrument eine andere Stellung yeben. In Fig. 1 steht der Stift $S$, der in dem Ringe $R^{\prime}$ befestigt ist, gerade nach oben. Mit dem Stift $\mathrm{S}$ lässt sich sber dieser Ring in der verticalen Ebene des Ringes $R^{\prime \prime}$ in jeder Richtung drehen. Der Index i“ zeigt hierbei auf der Gradeintheilung $\mathrm{g}^{\prime \prime}$ das Maass dieser Drehung an, welche in Fig. $245^{\circ}$ beträgt. Offenbar hat sich mit dem Ringe $R$ auch die Axe $a^{\prime} a^{\prime}$ bewegt und wir sehen in Fig. 2 das Ophthalmotrop in dieser Stellung abgebildet, - ausserdem das Auge bereits um die in ihrer neuen Stellung befindlichen Axe $a^{\prime} a^{\prime}$ gedreht. Vor dieser Drehung wurden die Stäbchen, welche der Neigung des Stiftes S gefolgt waren, wieder vertical gestellt, wobei der Index $i^{0}$ bis za derselben Anzahl Grade gelangte wie der Index $i$ ". Der Stand der Stäbchen vergegenwärtigt den verticalen Meridian des Auges. Man kann sich also vorstellen, dass das Auge nicht mit dem Stift $\mathrm{S}$ im äussersten Ringe herumgedreht sei: es ist als ob das lebende, in der primären Stellung verharrende Auge sich nur vorgenommen hätte, schräg nach oben oder schräg nach unten zu sehen. In Fig. 2 ist, wie gesugt, die Bewegung nun bereits ausgeführt und ist die Fixationslinie durch Drehung um die Axe a'a nach rechts und oben gerichtet. Um wie viele Grade es um die Axe gedreht wurde, giebt wieder der Index $\mathrm{i}^{\prime \prime}$ an, welcher in Fig. 2 auf $45^{\circ}$ zeigt. Das Auge ist mithin, in dem hier gewählten Fall, un $45^{\circ}$ nach oben gedreht um eine Axe 
$a^{\prime} a^{\prime}$, welche $45^{\circ}$ von der Horizontalen abweicht. Dieser Axe kann durch Drehung des Stiftes $S$ jede gewünschte Richtung gegeben und dergestalt die Fixationslinie aus der primären Stellung nach allen Seiten gerichtet werden, immer um Axen, welche unveränderlich in derselben Fläche des Ringes $R^{\prime \prime}$ liegen und ungefähr mit dem Aequator des Auges zusammenfallen*). Das ist die Illustration des Listing'schen Gesetzes.

Aus der primären Stellung, welche wir durch das Verticalstellen der Stäbchen jedesmal auf's Neue bestiminen, bewegen wir die Fixationslinie stets in der Richtung nach oder $a b$ von dem Stifte $S$, der mit der priinären und secundären Stellung der Fixationslinie in dem, bei dieser Drehung unveränderlich seine Stellung behaltenden Meridian verbleibt. Offenbar müssen also auch die Nachbilder von Linien, die in diesem Meridian liegen, bei der Drehung zusammenfallen mit den Bildern von Objecten, welche in demselben Meridian liegen. So wird durch das Phaenophthalmotrop der Hel mholtz' sche Beweis für die Richtigkeit des Lis ting'schen Gesetzes veranschaulicht: hätten wir die Stäbchen die Richtung des Stiftes behalten lassen, so würden sie bei der Drehung um die Axe $a^{t} a^{\prime}$ in dem unbeweglichen Meridian geblieben sein.

Aber ist auch der verticale Meridian bei der Drehung vertical geblieben? Wir haben die Stäbchen eben deshalb vor der Drehung in die verticale Stellung gebracht, um darüber urtheilen zu können, und nun ergiebt sich in der That, dass der Meridian aufgehört hat, vertical zu stehen und deutlich nach der rechten Seite hinüberneigt, - gerade wie das Nachbild eines verticalen

*) Das Bewegungscentrum (der Drehpunkt) liegt ein wenig hinter dem Centrum des Auges: also liegt der Ring $R^{\prime}$ etwas hinter dem Aequator, in einer diesem parallelen Ebene. 
Bandes, wenn wir nach oben rechts sehen. So wird also durch das Phaenophthalmotrop zugleich die an den Nachbildern beobachtete Stellung des verticalen Meridians wiedergefunden.

Man kann nun ferner auch den Grad der Neigung des verticalen Meridians in Graden ablesen: Man braucht zu dem Ende nur zu beobachten, um wie viel Grade der Index $\mathrm{i}^{0}$ sich verschiebt, wenn die Stäbchen mit der Fixationslinie in eine und dieselbe verticale Ebene $\mathrm{zu}$ rückgebracht werden. Dies kann mit Genauigkeit geschehen, wenn wir, indem wir mitten durch den mit einem Fadenkreuz versehenen Axencanal des Auges blicken, die Stäbchen mit einem vertical aufgehängten Faden zusammenfallen lassen.

Um sich bei diesen Versuchen die Bewegung des eigenen Auges auch gut zu vergegenwärtigen, thut man am Besten, das Phaenophthalmotrop auf der Höhe seines Auges anfzustellen und nun ein oder das andere Auge gerade dahinter $z u$ bringen. Man kann dann bequem allen Bewegungen folgen, die Drehungsaxe sich dabei deutlich vorstellen und die Versuche mit Nachbildern damit verbinden.

Noch einmal weisen wir darauf hin, dass man alle Axen, um welche sich das Auge dreht, wenn es aus der primären in die secundäre Stellung übergeht, durch Drehen des Ringes $R^{\prime}$ in $R^{\prime \prime}$ erhält und diese deshalb alle in Aequator liegen. Es stehen also sämmtliche Axen lothrecht auf der Fixationslinie: folglich kann hierbei von einer Drehung um die Fixationslinie, von einer Raddrehung, keine Rede sein. Es ist eben, wie das Listing'sche Gesetz bei Rü te formulirt steht: ,eine Relation, vermöge welcher die auf die optische Axe projicirte Drehung $=0$ wird."

Die Frage ist also: in welchem Sinne spricht Helm holtz hierbei von einer Raddrehung? Auch diese Frage 
wird durch das Phaenothalmotrop vollkommen aufgeklärt. Helmholtz geht bei der Analyse der Augenbewegungen von einer festen, im Auge gelegenen Ebene aus, dem Netzhauthorizont, welcher, bei aufrechter Kopfhaltung, auf den unendlich entfernten Horizont gerichtet, mit der Fixationsebene zusammenfällt: es ist also der horizontale Meridian des Phaenophthalmotrops, wenu alle Indices auf $0^{0}$ zeigen (Fig. 1). Die Richtung, welche die Fixationsaxe wirklich durch die Drehung um eine schräge Axe (die Axe $a^{\prime} a^{\prime}$ in Fig. 2) erhalten hat, lässt nun Helmholtz, indem er von der primären Stellung ausgeht, durch zwei Drehungen zu Stande kommen, die beide am Phaenophthalmotrop zu ermöglichen sind: $1^{0}$ eine Drehung um die Queraxe $a^{\prime} a^{\prime}$ (Erhebungswinkel), wobei die Fixationslinie nach oben oder nach unten, und $2^{0}$ eine Drehung um die Axe a (Seitenwendungswinkel), wobei die Fixationslinie nach der Seite gerichtet wird. Die zweite Axe a a befindet sich im Ringe $R$, und aut dem Gradbogen g wird der Seitenwendungswinkel abgeleser, sowie der Erhebungswinkel auf dem Gradbogen $\mathbf{g}^{\mathbf{1}}$ : wir müssen bemerken, dass die Axe aa, welche lothrecht aut dem Netzhauthorizont steht, bei der vorhergehenden Drehung um die Axe $\mathrm{a}^{\prime} \mathrm{a}^{\prime}$, mit dem Horizont ihre Richtung verändert, dabei aber stets in einer verticalen Ebene bleibend. Hat man nun durch Drehung um die Axen $a^{\prime} a^{\prime}$ und a der Fixationslinie eine Richtung gegeben, gleich derjenigen, welche in Fig. 2 durch Drehung um die unter $45^{\circ}$ gestellte Axe $a^{\prime} a^{\prime}$, nach dem Listing. schen Gesetz, hervorgebracht ist, dann hat der verticale Meridian dabei eine andere Neigung angenommen. $\mathrm{Er}^{\circ}$ neigt noch mehr nach rechts hinüber. Um also die Stellung zu erhalten, die das Auge bei der Drehung nach dem Listing'schen Gesetz wirklich annimmt, muss eine dritte Bewegung hinzugefügt werden, und zwar eine Drehung um die Fixationsaxe, d. h. - eine Raddrehung, - 
in dem angenommenen Falle von der rechten nach der linken Seite.

Diese Analyse bestimmt genau die Stellung der Augen und der Fixationslinien in Bezug zum Kopfe und ist zur mathematischen Behandlung sehr geeignet. Aber man betrachte sie als eine mathematische Fiction, nicht als eine physiologische Realität. Bei der Drehung um eine schräge Axe, nach dem Listing'schen Gesetz (Fig. 2), kommt ebensowenig Raddrehung, d. h. Drehung um die Fixationsaxe vor, als bei den aufeinanderfolgenden Drehungen um die Axen $a^{\prime} a^{\prime}$ und aa (wenn man vou der Stellung in Fig. 1 ausgeht): immer sind es Drehungen um eine Axe, senkrecht zur Fixationsaxe. Raddrelungen haben wir nur anzunehmen bei Bewegungen, die von dem Donders'schen und Listing'schen Gesetz abweichen.

Inzwischen kann man am Phaenophthalmotrop die Raddrehung im Sinne von Helmholtz für jede Stellung veranschaulichen und in Graden bestimmen. Man stelle durch Drehung am Stift $S^{\prime}$ die Axe $a^{\prime} a^{\prime}$ nach Wunsch ein (abzulesen auf $g^{\prime \prime}$ ), richte $k \mathrm{k}$ vertical und drehe das Auge um a'a', soviel man für gut befindet (abzulesen auf $8^{\prime}$ ). Nun bestimme man (s. pag. 163), um wie viele Grade der verticale Meridian, in Folge dieser Drehung nach dem Listing'schen Gesetz, nach der einen oder anderen Seite überneigt und beobachte durch den Axencanal, auf welchen Punkt im Raum die Fixationslinie gerichtet ist. Darauf bringe man das Auge wieder in die primäre Stellung, den Stift gerade nach oben, die Axe a'a' also horizontal, stelle $\mathrm{kk}$ vertical und richte nun, indem man durch den Axencanal blickt und gleichzeitig um die Axen aa und a'a dreht, die Fixationslinie auf denselben Punkt im Raum, als beim ersten Versuch. Nun überzeugt man sich sofort, dass $\mathrm{kk}$ mehr von der verticalen Stellung abgewichen ist, als im ersten Fall, und bestimme 
die Stellung wieder durch Herstellung und Ablesen auf $\mathrm{g}^{0}$. Der Unterschied der Neigung in den beiden Fällen, der hiermit gefunden ist, ist die Axendrehung von Helmholtz: die Ziffern entsprechen der durch Helmholtz*) gegebenen Tabelle. - Damit ist zugleich aufgeklärt, was von Vielen nicht begriffen wird, dass, während beim Fixiren, z. B. nach rechts oben, der verticale Meridian gemäss der Angabe der Nachbilder, nach rechts hinüberneigte, Helm hol tz dennoch von einerRaddrehung von rechts nach links sprechen konnte, denn, wie der Vergleich der beiden eben genannten Versuche lehrt, falls die secundäre Richtung der Fixationsaxe nur durch Drehung um die Axen aa und $a^{\prime} a^{\prime}$ erreicht worden wäre, würde der verticale Meridian eine noch stärkere Neigung nach rechts hinüber angenommen haben.

Wir haben jetzt noch zu sehen, wie Helmholtz die durch inn in die Analyse aufgenommene Raddrehung aus den Nachbildern direct bestimmte. Der Sinn und die Berechtigung dieser Analyse werden dann noch deutlicher werden.

Helmholtz geht, wie bereits bemerkt, von einer festen Ebene im Auge, der Netzhautebene, aus. Ein horizontales Band, in derselben Ebene auf einer senkrechten Wand ausgespannt, formirt sein Bild auf der Netzhaut in dem Horizont. Beim Drehen des Netzhauthorizontes nun um die Axe $a^{\prime} a^{\prime}$, fährt er fort die Wand in horizontalen Linien zu schneiden und das Nachbild wird mithin auf jeder Höhe mit horizontalen Linien auf der Wand zusammenfallen: das Phaenophthalmotrop macht dieses sogleich anschaulich, wenn man den Stäbchen durch Umdrehung von $r$ eine horizontale Richtung $k^{\prime}{ }^{\prime}{ }^{\prime}$ gegeben hat. Aber jetzt drehe man um die Axe a a (Seitenwendungswinkel): die Stäbchen $k^{\prime} k^{\prime}$ verlassen dann die horizon-

*) Physiologische Optik. S. 467. 
tale Stellung (und würden, wenn der Erhebungswinkel $90^{\circ}$ betragen könnte, sich selbst in einer verticalen Ebene drehen und für jeden Grad der Drehung also einen Grad von der horizontalen Richtung abweichen); aber, durch den Axencanal blickend, bemerkt man, dass sie, auf die Wand projicirt, doch vollkommen mit den darauf gezogenen horizontalen Linien zusammenfallen. Der Netzhauthorizont bleibt nämlich bei der Drehung um aa beständig in derselben Ebene, denn a steht lothrecht darauf und er muss also die Fortsetzung der horizontalen Linie, die auch in derselben Ebene liegt, aufnehmen. Das Phaenophthalmotrop macht uns dies anschaulich. Inzwischen ergiebt sich, dass das Nachbild eines horizontalen Bandes beim Blick seitwärts nach oben oder unten, nicht mehr mit horizontalen Linien auf der Wand zusammenfällt, dass nämlich, im Verhältniss zu diesen, der Netzhauthorizont, beim Blick nach oben, in entgegengesetztem Sinne gedreht ist: deshalb, sagt Helmholtz, hat eine Raddrehung in entgegengesstztem Sinne Statt gefunden, und das ist eben die Raddrehung, welche bei seiner Analyse postulirt wird.

In der That überzeugt man sich leicht, dass, während beim Blick schräg nach oben das Nachbild eines verticalen Bandes nach derselben Seite hin von verticalen Linien abgewichen ist, das Nachbild eines horizontalen Bandes in entgegengesetzter Richtung von horizontalen Linien abweicht. Die Fig. 1.

beiden rechtwinklig auf einander stehenden schwarzen Linien (Figur 1. $\mathrm{cv}$ und $\mathrm{ch}$ ), welchè zwei hellfarbige Bänder auf der Wand vorstellen, die in der primären Stellung in c fixirt sind und im Netzhauthorizont liegen, produ-

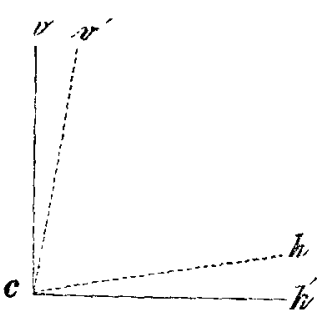
ciren, nach rechts und oben 
projicirt, ihre Nachbilder in der Richtung der beiden punktirten Linien $c \mathrm{v}^{\prime}$ und $c \mathrm{~h}^{\prime}$ : das Nachbild des verticalen Bandes ist also, beim Blick nach oben, nach derselben Richtung, das des horizontalen aber nach entgegengesetzter Richtung hin abyewichen. Die Erklärung davon liegt auf der Hand. Eine verticale Linie fält zusammen mit jeder anderen verticalen Linie, worauf sie projicirt wird, welche Stellung sie im Verhältniss zu einander und zum Auge auch immer einnehmen mögen. Mit horizontalen Linien ist es ganz anders: eine horizontale Linie, die sich von uns entfernt, wird absteigend gesehen, wenn sie über, aufsteigend aber, wenn sie unter unserem Auge gelegen ist. In einer Projection des Gesichtsfeldes, wie uns dies jede Zeichnung, jede Photographie lehrt, sind alle verticalen Linien vertical und bekommen alle horizontalen eine Neigung, die abhängig ist von der Richtung und Höhe, welche die Linien im Verhältniss zum Auge im Raum einnehmen. So wird nun eine horizontale Linie, die oberhalb unseres Auges auf einer senkrechten, der Gesichtsfläche parallelen, Wand gezogen ist, als sich senkend, gesehen und es ist gegenüber dieser Projection, dass das Nachbild einer horizontalen Linie, die in der Ebene der Primärstellung und also nicht als sich senkend gesehen wurde, eine $A b w e j-$ chung zeigt, wie in Fig. 1 von $h$ nach $h^{\prime}$, - im Gegensatz zu derjenigen eines Nachbildes eines verticalen Bandes von $\mathbf{v}$ nach $v^{\prime}$. Man projicire das Nachbild einer horizontalen Linie auf eine Ebene, worauf die Punkte einer horizontalen Linie sich sämmtlich in gleichem $\mathrm{Ab}$. stande vom Auge befinden und der Unterschied der Abweichung von horizontalen und verticalen Nachbildem rällt weg. Am vollkommensten würde diese Bedingung erfüllt sein, wenn sich das Auge im Mittelpunkt einer Kugel oder in der Axe einer cylindrisch gebauten Kammer befände und auf die Wand dieser Kugel oder dieser 
Kammer projicirte.*) Aber es ist schon ausreichend, wenn die verticale Ebene, in welcher die Fixationslinie bei der Projection liegt, lothrecht zur Wand stent. Hat man also zwei verticale Wände, wovon $w$ und $w^{t}$ (Fig. 2) Durchschnitte sind, und fixirt das Auge o, in seiner primären Stellung, auf Fig. 2 .

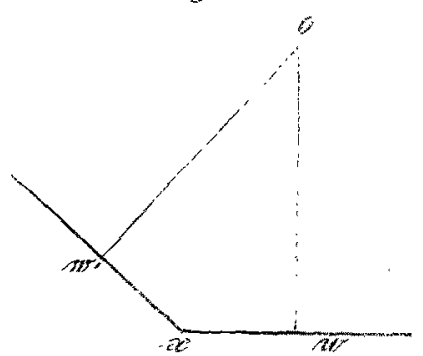
der Wand w den Kreuzungspunkt c der Ränder vh, dann zeigt die Projection der Nachbilder auf der Wand $w^{\prime}$. auf welcher die durch die Fixationslinie $o w^{*}$ gehende verticale Whene lothrecht steht, die Abweichung beider in gleichem Sinne (Fig. 3) Und steht man in Fig. 3. einer viereckigen Kanmer, so dass der Winkel $x$ ein rechter Winkel ist, dann ist bei der Projection auf $w$ nahe beim Winkel $x$, die Abweichung von $h$ in gleichem Sinne selbst grösser als die von $v$, weil horizontale Linien, auf $w^{\prime}$ gezogen, von dem Winkel aus-

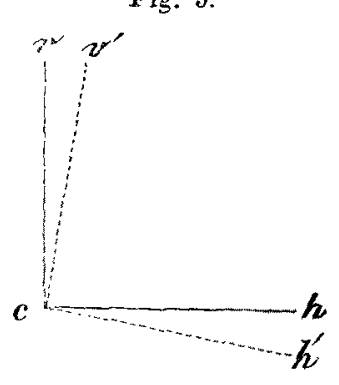
gehend sich dann dem Auge nähern. Sehr schlagend ist der Versuch, wenn man das Nachbild eines ziemlich langen horizoutalen Bandes, dessen Mitte man in der primären Stellung fixirt hat, in einer solchen viereckigen Kammer in den Winkel x projicirt, so dass es theils auf $w$, theils auf $\mathrm{w}^{\prime} \mathrm{zu}$ fallen kommt: in $\mathrm{w}$ steigt dann das Nachbild relativ $z u$ der horizontalen Linie, um sich auf w stark zu senken.

* Die beschreibenden und die horizontalen Linien eines Cylinders, dessen Axe durch den Mittelpunkt einer Kugel geht, fallen zusammen mit den Meridianen und Parallelen dieser Kugel, vom Mittelpunkte aus gesehen. 
Was hier die Nachbilder zeigen, kann man nun auch mit dem Phaenophthalmotrop veranschaulichen. Die Versuche kann man gesondert anstellen bei verticalen Stäbchen $\mathrm{k} k$ und horizontalen $\mathrm{k}^{\star} \mathrm{k}^{\star}$, oder auch gleichzeitig, indem man gleiche Stäbchen in $\mathbf{u}$ und $\mathbf{u}^{\prime}$ einschraubt, wodurch man ein Kreuz erhält (wie in Fig. 1), oder indem man sich auf zwei Stäbchen $k$ und $k^{\star}$ beschränkt, welche rechtwinkelig auf einander stehen. Durch den Axencanal blickend, projiciren sich dann die Stäbchen anf jeder Wand in derselben Richtung, wie sich dort die Nachbilder zeigen.

Der Leser wird leicht eingesehen haben, dass man, um sich die Bewegungen des Auges vorzustellen, nach Gutdünken mit mir vom verticalen Meridian oder mit Helmholtz vom Netzhauthorizont ausgehen kann. Der verticale Meridian scheint nur insofern vorzuziehen, als bei dem gänzlichen Zusammenfallen aller auf einander projicirten verticalen Linien, unabhängig von ihrer Stellung relativ zum Auge, die Vorstellung viel einfacher und bequemer sein dürfte.

Bezüglich des Phaenophthalmotrops als Instrument, habe ich noch eins und das andere hinzuzufügen. Es wird in grossem und in kleinem Modell gefertigt, ersteres besonders geeignet zur Demonstration.

Es besteht ganz aus Messing, im kleinen Modell der eigentliche Augapfel aus Palmholz. Es steht auf einer messingenen Stange, welche ein- und ausgeschoben werden kann, um es nach der Höhe des Auges des Beob. achters, der durch den Axencanal sehen will, reguliren zu können und ruht auf einem breiten Fuss. An Stelle des Axencanals kann man vorn eine Linse einfügen und hinten ein mattes Glas, worauf ein Kreuz gezeichnet ist, mit welchem man die Stellung der sich darauf formirenden dioptrischen Bilder von verticalen und horizontalen 
Linien, in verschiedener Richtung gesehen, vergleichen kann.

Es werden auch einfachere Phaenophthalmotropen angefertigt, bei welchen nämlich die Drehung um die Axe a fehlt und mithin nur die Bewegungen nach dem Listing'schen Gesetz ausgeführt werden können, nicht die Analyse dieser Bewegungen nach Helmholtz. Diese Vereinfachung hat den Vortheil, dass der Augapfel nun in dem innersten Ring $\mathrm{R}$ um die Fixationsaxe drehbar wird, was uns in den Stand setzt, nach Drehung der Stange $S^{\prime}$, stets einen und denselben festen verticalen Meridian des Auges wieder in die verticale Stellung zu bringen, sodass man nun auch feste Anheftungspunkte der Muskeln auf dem Bulbus angeben kann. Soweit es nöthig ist, kann dies dazu dienen, um bei jeder Augenstellung sich ihre Lage und damit den Antheil, welchen sie an der Bewegung nehmen, besser zu vergegenwärtigen.

Das Phaenophthalmotrop giebt die Bewegungen, sowie die Gesetze von Donders und von Listing sie mit sich bringen. Diesen aber, wie bereits zu Anfang bemerkt wurde, gehorcht das Auge nicht vollkommen.

Erstens ist es bekannt, dass schon in der primären Stellung die Bilder verticaler Linien, die auf den beiden Netzhäuten gebildet werden, nach aussen nicht vollkommen auf einander projicirt werden, woraus folgt, dass die verticalen Meridiane bei dieser Stellung die beiden Netzhäute nicht in vollkommen correspondirenden Punkten schneiden. Es bedarf keiner complicirten Instrumente, um sich hiervon zu überzeugen. Es genügt, ein Prisma mit der brechenden Kante nach oben vor das eine Auge zu halten: eine verticale Linie zeigt dann eine kleine Knickung an der Stelle, wo das Sehen mit dem einen in das Sehen mit dem anderen Auge übergeht. Aber auch eines Prismas bedarf man nicht einmal. Fixirt man in der 
primären Stellung einen verticalen Faden, welcher in grossem Abstande aufgehängt sein möge, dann bemerkt man deutlich genug, dass er nach oben und nach unten in Doppelbildern auseinander weicht. Schiebt man bei stets geöffneten Augen nun abwechselnd einen kleinen schwarzen Schirm (oder zur Noth die Hand) vor das eine und vor das andere Auge, dann überzeugt man sich, dass die Bilder zu einander überneigen. Bei rascher Abwechselung, dann und wann auch einen Augenblick mit beiden Augen sehend, kann man recht wohl dafür sorgen, dass das Auge hinter dem Schirm in guter Fixation bleibt und, sobald es frei wird, sich nicht nach der Seite hin zu drehen braucht, was die Beurtheilung weniger rein machen würde. Ganz fort fällt dieser Cebelstand, wenn man, indem man den Faden mit beiden Algen fixirt, sich darauf beschränkt, das eine Auge zu verdecken, wobei, obwohl der Effect nur halb so gross als bei abwechselndem Bedecken beider Augen ausfällt, der Faden schon deutlich genug ein wenig nach der entgegengesetzten Seite hinüberneigt, - eine Vorstellung, welche, einmal entstanden, von selbst nicht so rasch schwindet. Die gleichviel zu 'einander überneigenden Bilder verbinden wir nun beim binocularen Sehen zu einer verticalen. Man kann deshalb die Versuche mit Nachbildern wohl binocular ausführen, allein besser ist es doch, besonders bei Convergenz, das eine Auge verreckt zu halten. Bei der Convergenz entsteht, wie Meissner und Volkmann zeigten, eine stärkere Neigung der verticalen Bilder gegen einander: das ist ein ersten Abweichen von den allgemeinen Gesetzen. Sie sind ferner, wie Helmholtz bewies, nicht vollkommen gültig für die äussersten Grenzen des Fixationsfeldes, wo Unregelmässigkeiten nicht fehlen, und unlängst hat Javal in Astigmatikern, bei seitlicher Neigung des Kopfes, eine kleine Axendrehung beobachtet 
"de sorte que", wie Helmholtz sich ausdrückt, "la position de l'oeil n'est pas indépendante de celle de la tête aussi rigoureusement que l'affirme la loi de Donders*)."

Alle diese Abreichungen müssen ihre Auflösung und Erklärung finden in dem so verwickelten Problem von der Entstehung unserer Vorstellungen aus den Eindrücken beim binocularen Sehen.

Endlich hat sich ergeben, dass der myopische Bau des Auges - allerdings ein krankhafter - bezüglich der Gesetze, die wip behandelten, sowie auch in mancher anderen Beziehung, kleine Abweichungeu mit sich bringt, welche, besonders aus dem Gesichtspunkt der Accommodation von Organen nach dem durch das Bedürfniss bestimmten Gebrauch, unsere ganze Aufmerksamkeit verdienen.

Zum Schlusse noch ein Wort über die Untersuchungen der Bewegungen bei paralytischen Störungen der Muskeln. Wir betreten hiermit ein Gebiet, auf welchem der schaffende Geist Albrecht von Graefe's**) sich mit Vorliebe bewegte und seinen Nachfolgern wenig zu thun ubriggelassen hat. Aus den Abweichungen der

*) Vgl. Helmholtz Optique physiologique. p. 671. Paris, 1867. traduite par Emile Javal et N. W. Klein. Wenn Javal an einer anderen Stelle (Astigmatisme, of Wecker, Traité théorique et pratique des maladies des yeux. T. II. p. 828. Paris, 1869), anf Grund einer geringen Axendrehung, die Hueck'sche Theorie wieder in's Leben ruft, so muss ich ihn ersuehen, sich daran erinnern zu wollen, dass zu Folge dieser Theorie, bis zu einer Neigung von 25 oder $28^{\circ}$, die Neigung des Kopfes völlig dureh Axendrehung compensirt werden würde, sodass die vertiealen Meridiane ihre Richtung unverändert innehalten würden, und sich ferner zu bemühen, die Experimente zu wiederholen, wodurch sie vor vielen Jahren bereits gefallen ist (Holländisehe Beiträge. 1846. S. $105 \mathrm{ff}$, und bes. S. 334).

**) Vgl, dessen klassische AbhandIungen im Archir f. Ophthalmol und seine Symptomenlebre der Augenmuskellähmungen. Berlin. 1867 
Bewegung und aus der Stellung der Doppelbilder, bei jeder Stellung der Augen, genau festgestellt und scharfsinnig analysirt, hat von Graefe die Diagnose abgeleitet, - die Art und den Grad jeder Störung mit Genauigkeit bestimmt. In gewöhnlichen Fällen entsprechen diese Untersuchungsmethoden vollkommen ihrem Zweek. Zwei Verhältnisse aber giebt es, worin sie uns mehr oder weniger im Stich lassen. Das eine ist das, wenn auf beiden Augen gleichzeitig paralytische Erscheinungen vorhanden sind, - was nicht zu den Seltenheiten gehört, - das andere, wo nur das eine Auge sein Sehvermögen conservirt hat. In letzterem Falle fällt der Vergleich von Doppelbildern von selbst weg, in ersterem macht die Complication die Analyse schwierig, wenn nicht unmöglich. In diesen Fällen nun glaube ich die Untersuchung der Nachbilder, die schon im Allgemeinen nicht verwerflich, anempfehlen zu dürfen. Besonders kommt der oben beschriebene Versuch (S. 159), wodurch Helmholtz das Listing'sche Gesetz bewies, in Betracht. Folgt das Nachbild, bei senkrechtem Kopf, nicht vollkommen der Richtung 'des ausgespannten Bandes, so ist dem Listing'schen Gesetz nicht Genügc geschehen: es ist Raddrehung vorhanden, und die Richtung der Abweichung giebt unmittelbar an, in welchem Sinne. Man kann den Versuch bei allen Richtungen des Bandes machen und die Nachbilder, sowohl nach unten als nach oben, in der durch schwarze Linien angedeuteten Richtung fortgehend projiciren lassen. So erhält man eine Anzahl von Angaben, welche in Vexbindung mit etwa wahrzunehmenden Störungen der Bewegung in jeder Beziehung genügen, um die Diagnose festzustellen.

Es ist wahr, bei doppelseitiger Muskelparalyse oder Blindheit des zweiten Auges verlieren wir die Mittel, um dem Kopfe genau die zur Untersuchung der Nach. 
bilder nöthige Stellung zu geben. Aber ich habe mich ibberzeugt, dass der kleine Fehler, der daraus resultiren kann, dem Werthe dieser Angaben keinen Abbruch thut, um so weniger, als der Schluss nicht aus der Abweichung bei einer einzelnen Richtung des Bandes, sondern aus dem Vergleiche der verschiedenen Abweichungen, bei verschiedenen Richtungen, wie solches nothwendig immer mit einer paralytischen Störung verknüpft ist, hergeleitet wird. 


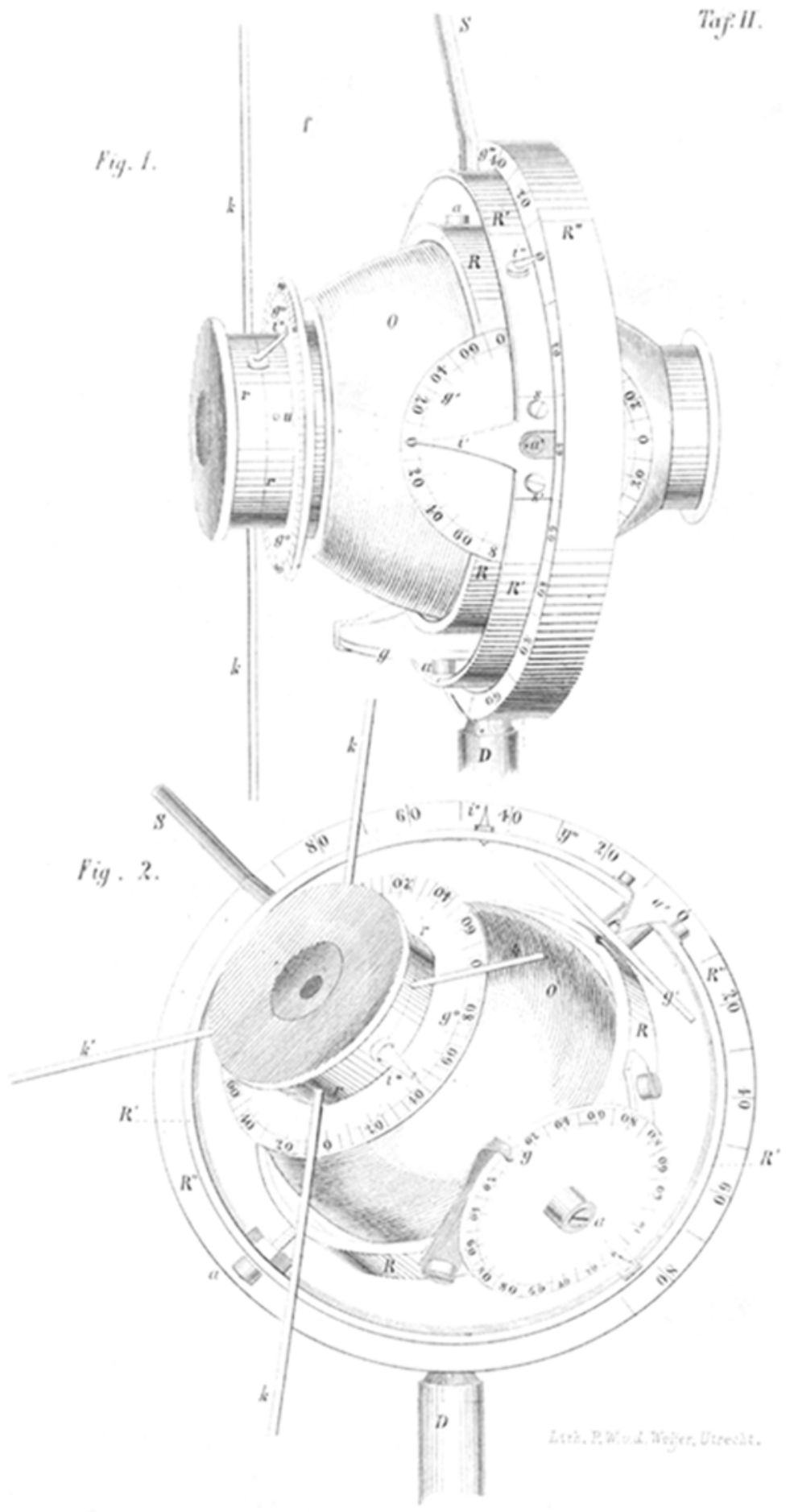

